\title{
Utilidad de una prueba serológica multiantigénica en el diagnóstico de tuberculosis
}

\author{
Claudia Marcela Castro ${ }^{1}$, Tania Bibiana Porras ${ }^{1}$, Martha Inírida Guerrero ${ }^{1}$, Clara Inés León ${ }^{1}$, \\ Maria Antonieta Mojica ${ }^{2}$, Martha Lara ${ }^{2}$, Maria Cristina Parada ${ }^{2}$, Clara Espitia². \\ ${ }^{1}$ Laboratorio de Micobacterias, Instituto Nacional de Salud, Bogotá, D.C., Colombia. \\ 2 Departamento de Inmunología, Instituto de Investigaciones Biomédicas, Universidad Nacional Autónoma \\ de México, México, D.F., México.
}

Introducción. La heterogeneidad en el patrón de reconocimiento antigénico observado en los enfermos infectados con Mycobacterium tuberculosis hace evidente la necesidad de contar con una técnica rápida y accesible para el diagnóstico de la enfermedad. La disponibilidad de pruebas que empleen diferentes antígenos podría ser de utilidad para aumentar la sensibilidad de la detección.

Objetivo. Evaluar el uso de una prueba multiantigénica (Mapia) de fácil manejo y evaluación visual en el diagnóstico serológico de la tuberculosis.

Metodología. Se estudiaron 66 sueros de pacientes con enfermedad tuberculosa comprobada y 47 sueros de individuos no tuberculosos, y se detectó la presencia de anticuerpos para 8 antígenos: 3 mezclas enriquecidas en antígenos y 5 antígenos recombinantes, incluidos en una matriz sólida cuya lectura se realizó cualitativamente. Los antígenos fueron evaluados por sus características de sensibilidad, especificidad y valores predictivos, confirmados mediante análisis de regresión logística del cual se obtuvo la razón de prevalencia utilizada para la selección de la combinación antigénica mas adecuada.

Resultados. Los valores de sensibilidad y especificidad de los antígenos individuales variaron entre $5 \%$ y $83 \%$, y $9 \%$ y $100 \%$, respectivamente; los valores de las mezclas enriquecidas fueron mejores que los valores presentados por los antígenos recombinantes. La combinación de varios antígenos mejoró notablemente los valores de sensibilidad hasta $81 \%$, pero en la mayoría de los casos se obtuvieron valores de especificidad menores al $57 \%$.

Conclusiones. Los resultados obtenidos sugieren que esta prueba podría ser útil como ayuda diagnóstica, especialmente para el tamizaje de poblaciones con sospecha de tuberculosis.

Palabras claves: tuberculosis/diagnósticos, tests serológicos, Mycobacterium tuberculosis, antígenos/uso diagnóstico

\section{Development of a multiantigenic serological test for tuberculosis diagnosis}

Background. The heterogeneity of the antigenic recognition pattern in tuberculosis patients makes evident the necesity of a rapid and accessible technique for tuberculosis diagnosis. One approach is the involvement of an antigen spectrum so that the sensitivity of the assay is improved.

Objective. The present work evaluated a multi-antigen printing immunoassay (MAPIA) for the serological diagnosis of tuberculosis.

Materials and methods. Sera were obtained from 66 patients with tuberculosis, verified clinically and bacteriologically and from 47 healthy individuals (control group). Sample sera were used for detection of antibodies against 3 enriched mixtures of proteins and 5 unique recombinant antigens. The antigens were presented in a solid matrix. Sensitivity, specificity and predictive values were evaluated and confirmed by a logistic regression analysis. A prevalence value was calculated and used for the selection of the best antigenic combination.

Results. The sensitivity and specificity values of individual antigens varied between $5-83 \%$ and $9-100 \%$. The enriched mixtures values were more accurate than those obtained with the 
recombinant antigens. Combinations of several antigens improved the sensitivity values up to the $81 \%$ level. In most cases, specificity values of $57 \%$ or less were obtained.

Conclusions. These results suggested that the multiantigenic test can be a useful screening tool, to be used in conjunction with the more definitive diagnostic tests.

Keywords: MAPIA, serologic test, Mycobacterium tuberculosis, antigen combination.

La tuberculosis es considerada a nivel mundial como una enfermedad reemergente que sigue siendo un grave problema de salud pública en muchos países, entre ellos, Colombia. Según la Organización Mundial de la Salud, gran parte de la población mundial ha tenido contacto con el bacilo tuberculoso y podría presentar la enfermedad en los próximos años (1). Se ha calculado que en el mundo se infectan alrededor de 8 millones de personas por año, de los cuales, 3 millones desarrollan la enfermedad anualmente. En Colombia, según el Ministerio de Salud, aparecen anualmente de 9.000 a 12.000 nuevos casos de tuberculosis (2). Por lo tanto, una estrategia importante para controlar la tuberculosis es la detección y el tratamiento temprano de los individuos afectados. Por esta razón, el desarrollo de métodos diagnósticos rápidos, accesibles y confiables constituyen una prioridad en la lucha contra la tuberculosis.

En la actualidad, la detección del agente etiológico de la tuberculosis realizada por baciloscopia y cultivo son las técnicas más usadas, a pesar de la limitada sensibilidad de la primera (3) y el tiempo prolongado que toma la segunda. Ante el desarrollo tecnológico en el área, se han producido e implementado métodos diagnósticos moleculares con ventajas y desventajas pero que, finalmente, continúan siendo costosos, requiriendo personal altamente capacitado e infraestructura sofisticada para su aplicación. Además, la toma de las muestras que han presentado aporte continúan siendo invasoras para el paciente.

Por otra parte, la posibilidad de obtener un diagnóstico serológico de tuberculosis ha sido

\footnotetext{
Correspondencia:

Clara Inés León, Avenida Calle 26 № 51-60, Bogotá, D.C., Colombia.

Teléfono: (571) 200926 y 2207700 , extensiones 436 o 498 ;

Fax: (571) 2207700 , extensión 255

cleon@hemagogus.in co.

Recibido: 08/07/04; aceptado: 16/12/04
}

explorada por muchos grupos de investigación. El primer intento por desarrollar una prueba serológica fue realizado por Arloing en 1898, quien creó una prueba de aglutinación con la cual no tuvo éxito (4). Posteriormente, se dieron avances importantes con la identificación y purificación de antígenos. Uno de estos fue la proteína de $65 \mathrm{kd}$ (5), el antígeno 5 (6) y sustancias no proteicas como el lipoarabinomanana y los glicolípidos fenólicos que, al final, resultaron ser inespecíficos de especie (7). Después de innumerables intentos se trató de buscar antígenos específicos de especie; los más estudiados fueron la proteína de choque térmico A60 (7-10), el antígeno de 38 kd (11-13) y el complejo antigénico 85 (14).

Se han estudiado diversos antígenos de manera individual y obtenidos de forma recombinante entre los cuales tenemos: la proteína de $38 \mathrm{kd}$ (15-18), el complejo antigénico 45/47 kd (19-21), el antígeno de $16 \mathrm{kd}$ (22), las proteínas ricas en glicina PE_PGRS $(23,24)$ y el antígeno de $30 \mathrm{kd}$ o $85 \mathrm{~b}(24, \overline{25})$.

Los resultados de los diferentes estudios han sido diversos y una de las principales limitaciones asociada con esta metodología ha sido la baja sensibilidad atribuida a la gran variabilidad del patrón de reconocimiento antigénico existente entre individuos (25), lo cual ha hecho que las pruebas que utilizan antígenos únicos no alcancen los valores de sensibilidad adecuados.

Estudios recientes llevados a cabo por Gennaro han demostrado que los valores de sensibilidad pueden mejorarse significativamente utilizando combinaciones de antígenos en lugar de utilizar un solo antígeno. Esta premisa no sólo es aplicable al diagnóstico serológico (26), sino también, a la evaluación de reacciones cutáneas, a las proteínas de $M$. tuberculosis, en individuos que tienen contacto con el bacilo (27).

La técnica Mapia descrita por Konstantin Lyashchenko (28) está basada en la inmovilización de una serie de antígenos dentro de una 
membrana de nitrocelulosa mediante microinyección semiautomática, seguida por el desarrollo de una técnica inmunocromógena que detecta la presencia de anticuerpos IgG, cuyo resultado se obtiene por evidencias visuales, sin requerimiento de equipos $(28,29)$.

La serología posee grandes ventajas: es una técnica simple, rápida, no invasora, que no requiere aislamiento ni cultivo del patógeno $y$, particularmente en el caso de Mapia, cuya lectura puede ser hecha visualmente por una persona entrenada, y que es posible llevarla a cualquier parte del mundo principalmente a países en desarrollo para contribuir con el diagnóstico de la tuberculosis extrapulmonar en la cual las ayudas son muy pocas y, en el caso de la tuberculosis paucibacilar pulmonar, en la que el diagnostico microbiológico no juega un papel relevante.

Por lo tanto, el principal valor de la serología se enfoca en el hallazgo de una técnica adecuada que contribuya en el diagnóstico de los casos de tuberculosis extrapulmonar, tuberculosis pulmonar en niños y adultos mayores en quienes se presenta la mayor incidencia de tuberculosis y entre los cuales se presenta dificultad en la obtención de la muestra y, además, de la poca respuesta a ayudas diagnósticas como el PPD en estos últimos (13).

El objetivo de este trabajo fue evaluar la utilidad de la combinación de antígenos puros y mezclas de fracciones enriquecidas en antígenos, inmovilizados en una matriz de polivinildifluoruro (PVDF), cuya reacción se pudiera detectar visualmente sin hacer uso de lectores de ELISA u otro equipo, metodología con posible aplicación en sitios de atención de salud de baja complejidad.

\section{Materiales y métodos}

\section{Sueros}

Se analizaron 113 sueros pertenecientes al banco de sueros del Laboratorio de Micobacterias del Instituto Nacional de Salud, en cuyas bases de datos se encuentra la información demográfica, geográfica, epidemiológica y clínica de los donantes de esos sueros. Las muestras seleccionadas se distribuyeron en tres grupos con características diferentes, así:
Grupo I: 33 sueros de pacientes con tuberculosis multibacilar demostrada bacteriológicamente por baciloscopia y cultivo, de los cuales, 28 tenían tuberculosis pulmonar y 5 tuberculosis extrapulmonar.

Grupo II: 33 sueros de pacientes con tuberculosis paucibacilar (baciloscopia negativa) confirmada por histopatología, cultivo o PCR, de los cuales, 19 tenían tuberculosis pulmonar y 14 tuberculosis extrapulmonar.

Grupo III: 47 sueros de individuos sin tuberculosis, descartada clínica y bacteriológicamente por baciloscopia, cultivo y PCR, de los cuales, 15 eran contactos de casos índices de tuberculosis, 14 convivientes de casos de lepra y 18 pacientes con otras enfermedades pulmonares diferentes a tuberculosis.

\section{Antígenos}

Se cultivó $M$. tuberculosis H37Rv en medio Proskawer Beck Youmans (PBY) durante 4 a 5 semanas. Los extractos proteicos, filtrado de cultivo y sonicado así como la fracción enriquecida del filtrado de cultivo (Fo) se obtuvieron como se ha descrito anteriormente $(18,21,30)$. Los antígenos individuales se obtuvieron como proteínas recombinantes expresados en Escherichia coli (Rv1759c o PGRS, Rv2031c o 16 kd y Rv1816c o 30 kd), Mycobacterium smegmatis (Rv0934 38 kd) y Streptomyces lividans (Rv1860 antígeno $45 / 47 \mathrm{kd}$ o Apa). Las proteínas obtenidas se purificaron según el método descrito previamente por Colangeli et al. (31) en el cual se asegura la eliminación de los diferentes contaminantes que pueden influir en la especificidad de la prueba, a la vez que comprueba paso a paso la obtención de la proteína de interés.

Tanto los extractos antigénicos como los antígenos individuales han sido evaluados previamente en su capacidad de generar una respuesta inmune humoral en tuberculosis (18-25).

\section{Preparación de las membranas}

La preparación de las membranas se llevó a cabo en el Departamento de Inmunología del Instituto de Investigaciones Biomédicas de la Universidad Nacional Autónoma de México. En una membrana 
de polivilnildifluoruro (Millipore) se colocaron manualmente $135 \mu \mathrm{l}(60 \mu \mathrm{g} / \mathrm{ml}$ diluidos en PBS) de cada uno de los antígenos recombinantes y $135 \mu \mathrm{l}(50 \mu \mathrm{g} / \mathrm{ml})$ de cada uno de los extractos proteicos usando un miniblotter 45 (Immunetics). Después de una hora de incubación, las proteínas fueron fijadas a la membrana mediante la adición de etanol al $50 \%$ y, finalmente, las membranas fueron cortadas perpendicularmente a las bandas impresas en tiras de $2 \mathrm{~mm}$ de ancho (figura 1). Se obtuvieron alrededor de 60 tiras por membrana con $0,15 \mathrm{mg}$ de antígeno recombinante y $0,1 \mathrm{mg}$ de los extractos antigénicos, aproximadamente.

\section{Prueba}

Las muestras de suero se diluyeron 1:100 en solución amortiguadora de bloqueo constituida por leche descremada al $2 \%$ en PBS-Tween $(10 \mathrm{mM}$ de solución amortiguadora de fosfato salino, $\mathrm{pH}$ $7,4,0,05 \%$ Tween 20 (Sigma P-3563), y con $1 \mathrm{ml}$ de esta solución se impregnó una tira la cual se llevó a incubación durante 1 hora. Posteriormente, se lavó con PBS-Tween, y las tiras se reincubaron durante 30 minutos con proteína A marcada con fosfatasa alcalina (Sigma P7488) diluida 1:2.000 en solución amortiguadora de bloqueo; concluido el tiempo de incubación se lavó con agua destilada.

La actividad de la enzima fue evidenciada ante la incubación de las tiras con 5-bromo-4-cloro-3indolil fosfato/fosfatasa de tetrazolio (Laboratorios Kirkegaard y Perry, Cat. 50-81-07) durante 5 min.
Finalmente, las tiras se lavaron con agua destilada y se pusieron a secar sobre papel filtro. Todo el procedimiento se llevó a cabo a temperatura ambiente con agitación manual permanente para asegurar la distribución homogénea de muestras y reactivos en la tira. Los resultados de la prueba se evaluaron visualmente; la presencia de bandas de cualquier intensidad se consideró como resultado positivo para la detección de anticuerpos $\lg$ (figura 2) (28).

\section{Análisis de resultados}

Mediante el programa Epilnfo 6.04, se calculó la sensibilidad (S), la especificidad (E) y los valores pronósticos de los antígenos individuales y en mezclas, con sus respectivos intervalos de confianza del 95\% (IC95\%), haciendo uso de tablas de contingencia.

La sensibilidad se calculó como el porcentaje de los resultados positivos obtenidos con el suero de pacientes tuberculosos. La especificidad se calculó como el porcentaje de resultados negativos obtenidos con sueros de individuos control negativo. El valor pronóstico positivo (VPP) se definió como la relación entre resultados verdaderamente positivos (pacientes tuberculosos) y el total de resultados positivos obtenidos, y el valor pronóstico negativo (VPN) como la relación entre los resultados verdaderamente negativos (individuos control no tuberculosos) y el total de resultados negativos.
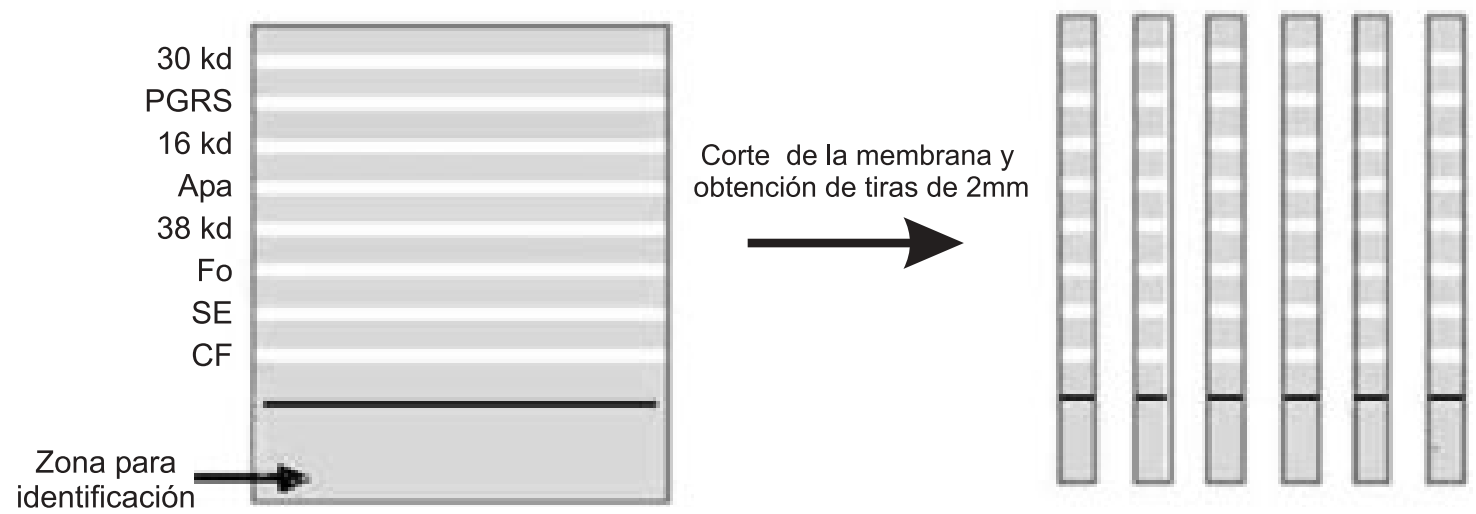

Figura 1. A la derecha se observa la membrana de PVDF luego de la impresión de los antígenos y a la izquierda, las tiras obtenidas por corte de la membrana en las cuales se tiene representación de cada uno de los ocho antígenos utilizados. 


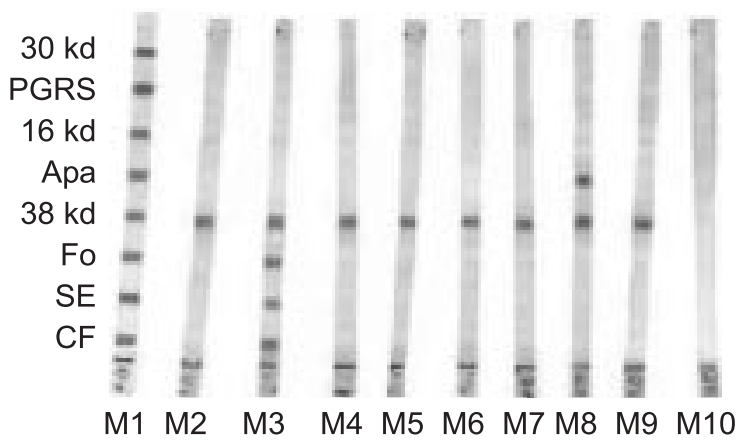

Figura 2. En la figura se observan los resultados obtenidos luego del desarrollo de la prueba serológica. Las bandas violeta indican respuesta positiva frente a cada antígeno, control positivo (M1); individuos enfermos (M2-M5, M8 y M9); individuos grupo control (M6, M7 y M10).

La selección de la combinación de antígenos se realizó por análisis conjunto de datos de sensibilidad, especificidad y valores pronósticos, con sus respectivos IC $95 \%$ y mediante el análisis de regresión logística, usando el programa MedCalc, versión 7.2.

\section{Resultados}

Se procesaron 113 sueros, 66 sueros de pacientes tuberculosos y 47 sueros de individuos control, y el análisis se realizó de 105 sueros debido a que se obtuvieron 8 resultados inespecíficos por la presencia de coloración de fondo que impidió la lectura.

\section{Antígenos analizados individualmente}

Se evaluó la sensibilidad y la especificidad de 8 antígenos de $M$. tuberculosis mediante Mapia con sueros de 60 pacientes con tuberculosis confirmada y 45 controles no tuberculosos (cuadro 1); se encontró que 54 sueros de pacientes con tuberculosis $(90 \%)$ reaccionaron, por lo menos, con un antígeno del panel. Entre los pacientes con tuberculosis confirmada que no respondieron a la prueba encontramos 2 adultos jóvenes positivos para VIH con tiempo de evolución de la tuberculosis menor a un mes y 4 adultos mayores de 64 años negativos para $\mathrm{VIH}$.

El antígeno de $38 \mathrm{kd}$ mostró una elevada sensibilidad $(83 \%)$ pero presentó también la más baja especificidad ( $9 \%$ ) en relación con los demás antígenos utilizados, por lo cual es poco útil. Después de éste, el antígeno con mayor respuesta fue el sonicado, el cual presentó una sensibilidad del $60 \%$ y especificidad del $56 \%$.

Los antígenos de $30 \mathrm{kd}$ y el PGRS tuvieron la mejor especificidad, $100 \%$ y $96 \%$, respectivamente, aunque presentaron muy baja sensibilidad. Los antígenos con mejor valor pronóstico positivo fueron $30 \mathrm{kd}$ y $16 \mathrm{kd}$ (cuadro 1).

Al evaluar por separado los resultados obtenidos entre los pacientes con tuberculosis pulmonar y extrapulmonar, se evidenció claramente que al descartar el antígeno de $38 \mathrm{kd}$, las proteínas sonicadas eran las más inmunorreactivas en ambos grupos con porcentajes de respuesta del $64 \%$ y $50 \%$, respectivamente (cuadro 1 ).

Vale la pena destacar la respuesta diferencial generada contra el antígeno de $16 \mathrm{kd}$ por parte de los pacientes con tuberculosis pulmonar ( $\mathrm{S}=24 \%$

Cuadro 1. Valores de sensibilidad, especificidad, valores pronósticos con sus intervalos de confianza del 95\%, para cada antígeno utilizado en Mapia en el total de muestras y distribuidos según el tipo de tuberculosis.

\begin{tabular}{|c|c|c|c|c|c|c|c|c|c|c|c|c|c|c|c|c|c|c|}
\hline \multirow{3}{*}{$\frac{\mathrm{Ag}}{\mathrm{CF}}$} & \multirow{2}{*}{\multicolumn{4}{|c|}{$\begin{array}{l}\text { Total } \\
\% \mathrm{E}\left(\mathrm{IC}_{95 \%}\right)\end{array}$}} & \multirow{3}{*}{$\begin{array}{r}\text { VPN } \\
50\end{array}$} & \multirow{3}{*}{$\begin{array}{c}\text { VPP } \\
64\end{array}$} & \multicolumn{4}{|c|}{ Pulmonar } & \multirow{3}{*}{$\frac{\text { VPN }}{62}$} & \multirow{3}{*}{$\begin{array}{c}\text { VPP } \\
58\end{array}$} & \multirow{2}{*}{\multicolumn{2}{|c|}{$\% \mathrm{~S}\left(\mathrm{IC}_{95 \%}\right)$}} & \multicolumn{4}{|c|}{ Extrapulmonar } \\
\hline & & & & & & & & $\mathrm{S}\left(\mathrm{IC}_{95 \%}\right)$ & $\%$ E (I & $\left(\mathrm{IC}_{95 \%}\right)$ & & & & & & $\% \mathrm{E}\left(\mathrm{IC}_{95 \%}\right)$ & VPN & VPP \\
\hline & 57 & $43,3-69,2$ & 58 & $42,2-72$ & & & 62 & $46-76$ & 58 & $46-76$ & & & 44 & $22,4-68,7$ & 58 & $42,2-72$ & 72 & 30 \\
\hline SE & 60 & $46,5-72,2$ & 56 & $40,1-70$ & 51 & 64 & 64 & $48-78$ & 56 & $40-70$ & 62 & 57 & 50 & $27-73$ & 56 & $40-70$ & 73 & 31 \\
\hline FO & 60 & $46,5-72,2$ & 51 & $36-66,1$ & 49 & 62 & 64 & $48-78$ & 51 & $36-66$ & 60 & 55 & 50 & $26,8-73$ & 51 & $36-66$ & 72 & 29 \\
\hline $38 \mathrm{kd}$ & 83 & $71,0-91,3$ & 9 & $2,9-22,1$ & 29 & 55 & 76 & $60-87$ & 9 & $3-22$ & 29 & 44 & 100 & $78-100$ & 9 & $3-22$ & 100 & 30 \\
\hline Apa & 22 & $12,5-34,5$ & 78 & $62,5-88,3$ & 43 & 57 & 21 & $11-37$ & 78 & $62-88$ & 51 & 47 & 22 & $7,4-48$ & 78 & $62-88$ & 71 & 29 \\
\hline $16 \mathrm{kd}$ & 38 & $26,4-51,8$ & 89 & $75,2-95,8$ & 52 & 82 & 24 & $13-39,8$ & 89 & $90-100$ & 58 & 74 & 50 & $27-73$ & 89 & $75-96$ & 82 & 64 \\
\hline PGRS & 5 & $1,3-14,8$ & 96 & $83,6-99,2$ & 43 & 60 & 5 & $0,8-17$ & 96 & 84-99 & 52 & 50 & 6 & $0,3-29$ & 96 & $8,4-99$ & 72 & 33 \\
\hline $30 \mathrm{kd}$ & 18 & $9,9-30,9$ & 100 & $90,2-100$ & 48 & 100 & 24 & $13-40$ & 100 & $90-100$ & 58 & 100 & 37 & $20-57$ & 100 & $90-100$ & 73 & 100 \\
\hline
\end{tabular}

CF: filtrado de cultivo, SE: sonicado, Fo: fracción enriquecida del filtrado de cultivo, Apa: complejo antigénico 45/47, 16 kd, PGRS: proteínas ricas en glicina y $30 \mathrm{kd}$ o antígeno $85 \mathrm{~B}$. 
y VPN $=58 \%$ ) y extrapulmonar con sensibilidad de $50 \%$ y VPN de $82 \%$ (cuadro 1 ).

Mediante análisis de regresión logística se determinó que el antígeno de 16 kd presentaba una mayor asociación con enfermedad en relación con los demás antígenos (OR=7,0; IC95\%: 1,7 a $28,3 ; p=0,0031)$.

\section{Evaluación de las combinaciones antigénicas}

Para la evaluación de la combinación antigénica no incluimos en las mezclas el antígeno de $38 \mathrm{kd}$ por su notable inespecificidad, ni los antígenos de 30 kd y PE_PGRS por su baja sensibilidad. Con los restantes cinco antígenos se llevó a cabo una serie de combinaciones para elegir la mejor mezcla antigénica posible, entre las cuales se seleccionaron aquéllas con mejores resultados (cuadro 2).

Al analizar las combinaciones observamos que la mejor combinación era la integrada por los antígenos filtrado de cultivo, sonicado, Apa y 16 kd (combinación 2) con sensibilidad del $73 \%$ y VPP de $67 \%$ (cuadro 2), datos confirmados mediante análisis de regresión logística $(O R=2,9$; IC95\%: 1,3 a 6,5; $p=0,01$ ) en comparación con las demás mezclas.

Al estratificar por grupos puede observarse que los pacientes del grupo 1 , microbiológicamente denominados multibacilares, tuvieron un porcentaje de respuesta mejor a la combinación antigénica 2. Los pacientes del grupo 2, paucibacilares, respondieron de igual forma a las combinaciones 1 y 2 (cuadro 2), notándose un incremento en la especificidad al usar la combinación 5 en este grupo.

La combinación total de antígenos (combinación 3) presentó una sensibilidad y especificidad de $73 \%$ y $47 \%$, respectivamente.

Al estudiar la respuesta dada por los pacientes con tuberculosis pulmonar y extrapulmonar a las combinaciones podemos notar que ambos responden de igual forma a las combinaciones 1 y 2 (cuadro 3).

\section{Valor discriminatorio de la prueba}

En nuestro estudio, la combinación 2 detectó el $81 \%$ de los pacientes con baciloscopia positiva y cultivo positivo, el $65,5 \%$ de los pacientes con baciloscopia negativa y cultivo positivo, y reconoce como positivos al $49 \%$ de los pacientes del grupo control. Teniendo como antecedentes que los individuos del grupo control incluidos en el estudio podrían estar infectados, se evidencia que la prueba no es capaz de discriminar entre los estados de enfermedad e infección.

\section{Discusión}

En nuestro estudio se observó que 6 de los pacientes tuberculosos no respondieron a ninguno de los antígenos, posiblemente debido al estado de anergia que puede presentarse en este tipo de pacientes: portadores de VIH y adultos mayores (13), por lo cual, no generaron respuesta alguna a los antígenos incluidos en la prueba.

Algunos autores afirman que el paciente responde a determinados antígenos según el tiempo de

Cuadro 2. Combinaciones antigénicas con mayores valores de sensibilidad, especificidad, valores pronósticos e intervalos de confianza del $95 \%$. Los resultados se presentan en la muestra total y estratificados por grupos.

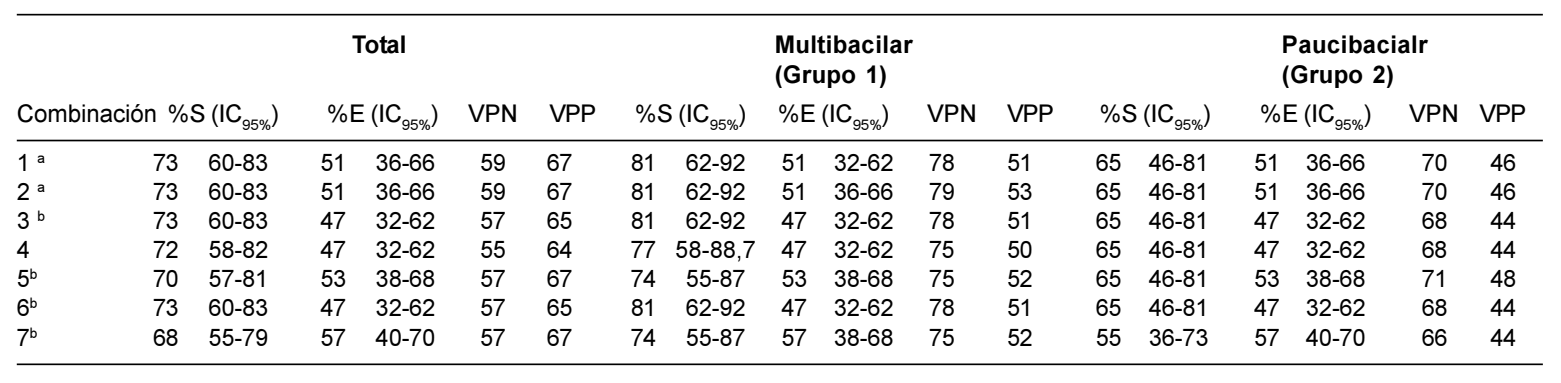

Combinaciones: $1=\mathrm{SE}+\mathrm{Apa}+16 \mathrm{kd} ; 2=\mathrm{CF}+\mathrm{SE}+\mathrm{Apa}+16 \mathrm{kd} ; 3=$ mezcla total; $4=\mathrm{CF}+\mathrm{FO}+\mathrm{Apa}+16 \mathrm{kd} ; 5=\mathrm{CF}+\mathrm{Apa}+16 \mathrm{kd} ; 6=\mathrm{SE}+\mathrm{FO}+\mathrm{Apa}+16 \mathrm{kd}$; $7=\mathrm{SE}+16$ kd Valor $\mathrm{p}$ de Mantel-Haenszel: ${ }^{\mathrm{a}}<0,011 ;{ }^{\mathrm{b}}<0,035$ 
evolución de la enfermedad, por ejemplo, los antígenos $38 \mathrm{kd}$ y $30 \mathrm{kd}$ se han detectado casi exclusivamente en estadios avanzados de la enfermedad (17).

$\mathrm{Al}$ igual que otros autores, nuestro estudio confirma que la especificidad del antígeno de 38 $\mathrm{kd}$ no es buena. Benjamin et al. (32) usaron el antígeno de $38 \mathrm{kd}$ en varios grupos de pacientes y se encontraron respuestas positivas en el $30 \%$ de los pacientes infectados con Mycobacterium kansassi, el $36 \%$ de infectados con Mycobacterium intracellulare, el $13 \%$ de pacientes con sarcoidosis, el $17 \%$ de individuos PPD positivos y el $5 \%$ de individuos PPD negativos. Por otra parte, la sensibilidad de $38 \mathrm{kd}$ tampoco es absoluta puesto que en este estudio sólo el $70 \%$ de los pacientes con tuberculosis activa respondieron a tal antígeno.

Con los resultados obtenidos podemos ver que la máxima sensibilidad alcanzada por los antígenos individuales fue tan sólo del $60 \%$ y estuvo dada por los sonicados y la fracción enriquecida del filtrado de cultivo, los cuales concentran la mayoría de los antígenos expresados por $M$. tuberculosis, razón por la cual la especificidad no resulta ser tan alta pues se sabe que las especies del género Mycobacterium comparten muchos de sus antígenos. Además, pudimos ver que entre los antígenos recombinantes únicos, la mayor respuesta se presentó frente a la proteína $16 \mathrm{kd}$, la cual hasta ahora es reconocida como específica de especie, a pesar de haber sido reconocida por
4 individuos del grupo control, lo cual podría atribuirse a hiperglobulinemias comunes en la enfermedad bronquial o por otras causas (22).

Una de las razones que se pueden atribuir a la variación en la sensibilidad es que los pacientes responden a diferentes antígenos y no existe un antígeno dominante en tuberculosis $(25,26)$.

Nuestro trabajo confirma lo que previamente han encontrado otros investigadores, en el sentido de que los antígenos únicos, no son la mejor alternativa para una prueba diagnóstica; razón por la cual la combinación de antígenos se propone como alternativa para aumentar la sensibilidad.

Las combinaciones 1 y 2 mostraron alta sensibilidad en la detección de tuberculosis multibacilar pulmonar y extrapulmonar y, en un grado un poco menor, en la detección de tuberculosis paucibacilar; en ésta, la especificidad fue tan sólo un poco superior al $50 \%$, lo cual demuestra su aplicabilidad como prueba de tamizaje.

De cualquier manera, si comparamos la sensibilidad de la baciloscopia en tuberculosis extrapulmonar $(10 \%$ a $40 \%)$ con la de estas combinaciones $(72 \%)$, vemos que en el mismo lapso de tiempo se tiene una prueba con un incremento significativo en la sensibilidad. Por otra parte, si tenemos en cuenta que el cultivo en tuberculosis extrapulmonar tiene una sensibilidad alrededor del $30 \%$, se debe considerar el sustancial ahorro de tiempo en la obtención del

Cuadro 3. Respuesta generada contra las combinaciones por los pacientes tuberculosos con enfermedad pulmonar y extrapulmonar.

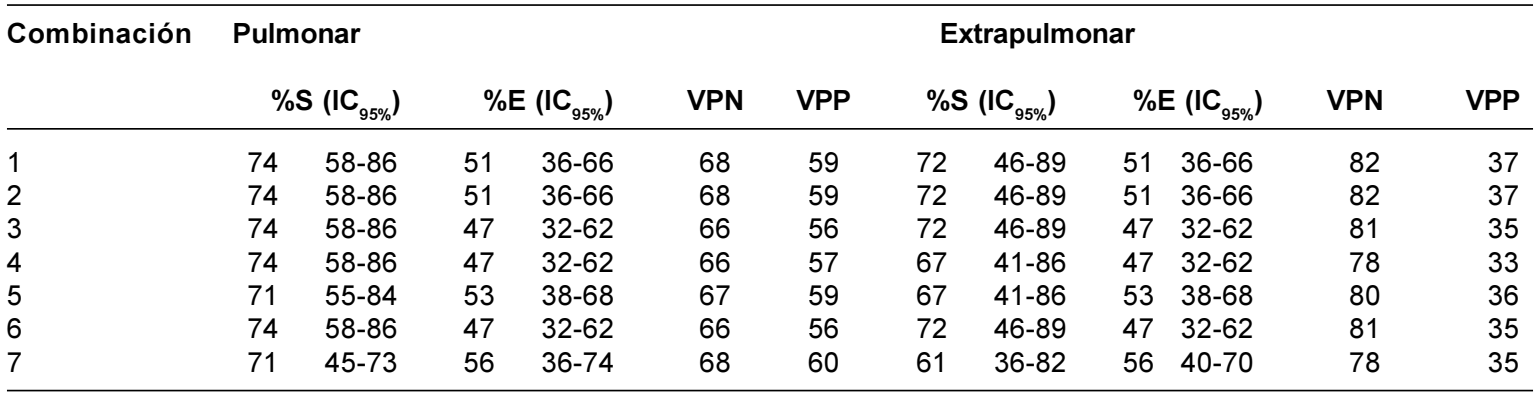

Combinación: $1=\mathrm{SE}+\mathrm{Apa}+16 \mathrm{kd}$; $2=\mathrm{CF}+\mathrm{SE}+\mathrm{Apa}+16 \mathrm{kd}$; $3=$ mezcla total; $4=\mathrm{CF}+\mathrm{FO}+\mathrm{Apa}+16 \mathrm{kd}$; $5=\mathrm{CF}+\mathrm{Apa}+16 \mathrm{kd} ; 6=\mathrm{SE}+\mathrm{FO}+\mathrm{Apa}+16 \mathrm{kd} ; 7=\mathrm{SE}+16 \mathrm{kd}$ 
resultado. Además, al comparar la serología Mapia, en particular el antígeno de $16 \mathrm{kd}$ ( $\mathrm{S}=50 \%$, $\mathrm{E}=89 \%$ ), con otras ayudas diagnósticas como la adenosindeaminasa (ADA) ( $\mathrm{S}=22 \%$ y $\mathrm{E}=69 \%$ ) (Guerrero MI, Naranjo N, Chaparro P, Matyas RE, Pacheco D, Latorre $\mathrm{P}$ et al. La reacción en cadena de la polimerasa para el diagnóstico de tuberculosis extrapulmonar. Biomédica 2000;22(Supl):21-2) entre el grupo de pacientes extrapulmonares, podemos destacar la utilidad de dicha serología como ayuda diagnóstica en estos casos.

Por lo tanto, debido a la facilidad, rapidez, bajo costo y poco riesgo en la manipulación, la prueba Mapia que contiene la combinación 2 constituye una alternativa importante para escoger a los individuos que deben ser sometidos a estudios más costosos, demorados y complicados, en la cual el antígeno de $16 \mathrm{kd}$ garantiza una elevada especificidad entre el grupo de pacientes extrapulmonares. Además, sugiere la posibilidad de instaurar quimioterapia prontamente en los casos de alto riesgo como las tuberculosis extrapulmonares, principalmente la meningitis tuberculosa en la cual no es posible esperar el resultado del cultivo.

Como ya se ha demostrado que en el periodo comprendido entre la consulta y el diagnóstico se pierden alrededor del $40 \%$ de los casos, la aplicación de esta metodología contribuiría a la disminución de tal pérdida (33).

La combinación seleccionada fue escogida porque presentaba los mejores valores de S, E, VPP, VPN y OR, en la cual se logró aumentar la sensibilidad, razón última del estudio. Sin embargo, no se logró discriminar entre infección y enfermedad pues se captó el $49 \%$ de los individuos control, lo cual constituye una gran limitación de la prueba, ya que en países como el nuestro en el que la prevalencia de tuberculosis y de lepra son altas, $y$ hay presencia de infecciones micobacterianas causadas por micobacterias ambientales, se hace necesario diferenciar entre infección y enfermedad tuberculosa. A este respecto, existen estudios que muestran un $49 \%$ de reactividad en pacientes con lepra sin enfermedad tuberculosa (11) frente a 38 $\mathrm{kd}$, uno de los principales componentes de las proteínas del filtrado de cultivo, incluido en la combinación 2.

Este estudio nos demuestra que la consecución de una prueba serológica que sea diagnóstica para tuberculosis no es fácil. Con base en los resultados de este estudio proponemos la serología Mapia utilizando la combinación 2 como prueba de tamizaje mas no como prueba diagnóstica confirmatoria.

Después de esta primera fase del trabajo creemos que se hace necesario realizar un estudio clínico utilizando las metodologías tradicionales y, además, la serología de Mapia en el cual se siga a la población para determinar cuál es el verdadero aporte de esta prueba serológica, enfocando principalmente la utilidad como ayuda diagnóstica en los casos de tuberculosis extrapulmonar.

\section{Agradecimientos}

A Franklin Prieto por su colaboración en el análisis estadístico y a Carlos Arturo Hernández por su colaboración en la escritura del artículo.

\section{Conflicto de intereses}

Los autores manifiestan que no existe ningún conflicto de interés.

\section{Financiación}

El presente estudio se realizó gracias a la financiación del Instituto Nacional de Salud, Colombia, proyecto INS-CTI 39 de 2002 y la Comunidad Económica Europea, CEE, programa INCO-DEV, proyecto ICFP 500 A4PR02 CA-INCO ICA 4-2000-10054.

\section{Referencias}

1. World Health Organization. Report on the tuberculosis epidemic. Geneve, Switzerland: World Health Organization; 1996.

2. Victoria J. Situación epidemiológica de la tuberculosis en Colombia. Revista de la Facultad Ciencias de la Salud de la Universidad del Cauca 1999;1:27-31.

3. Toman K. Sensitivity, specificity and predictive value of diagnostic test. Bull Int Union Tuberc 1981;56:18-28.

4. Daniel TM, Debanne SM. The serodiagnosis of tuberculosis and other mycobacterial diseases by enzime-linked immunosorbent assay. Am Rev Respir Dis 1987;135:1137-51. 
5. Shinnick JM, Vadkin MH, Williams JL. The Mycobacterium tuberculosis $65 \mathrm{kd}$ protein is a heat shock protein is a heat shock protein which corresponds to common antigens and to the $E$. coli $\mathrm{EL}$ protein. Inf Immun 1988;6:446-51.

6. Balestrino E, Daniel T, De Latini MDS, Latini OA, Ma Y, Scocozza JB. Serodiagnosis of pulmonary tuberculosis in Argentina by enzyme-linked immunosorbent assay (ELISA) of IgG antibody to Mycobacterium tuberculosis antigen 5 and tuberculin purified protein derivate. Bull Who 1984;62:755-62.

7. Maes R. Clinical usefulness of serological measurements obtained by antigen 60 mycobacterial infections: development of a new concept. Klin Wochenschr 1991;69:696-709.

8. Kaustová J. Serological IgG, IgM and IgA diagnosis and prognosis of mycobacterial diseases in routine practice. Eur J Med Res 1999;1:393-403.

9. Singh P, Baveja CP, Talukdr B, Kumar S, Mathur MD. Diagnostic utility of ELISA test using antigen A60 in suspected cases of tuberculous meningitis in pediatric age group. Indian J Pathol Microbiol 1999;42:11-4.

10. Gupta A, Kumar V, Xess A, Sharma H, Shahi S. Role of enzyme linked immunosorbent assay in the diagnosis of suspected cases of genitourinary tuberculosis. Indian J Pathol Microbiol 1999;42:307-9.

11. Harboe M, Wiker H. The $38 \mathrm{kDa}$ protein Mycobacterium tuberculosis: a review. J Infect Dis 1992;166:874-84.

12. Devi U, Ramalingam B, Brennan PJ, Narayanan PR, Raja A. Specific and early detection of IgG, IgA and IgM antibodies to Mycobacterium tuberculosis $38 \mathrm{kDa}$ antigen in pulmonary tuberculosis. Tuberc 2001;81: 249-53.

13. Chan ED, Heifets L, Iseman MD. Immunologic diagnosis of tuberculosis: a review. Tuber Lung Dis 2000; 80:131-40.

14. Jae-Hyun L, Jeong-Kyu P, Eun-Kyeong J, ChangHwa S, Dullei M, Young-Ja $S$ et al. Purification and immunoreactivity of three components from the 30/32kilodalton antigen 85 complex in Mycobacterium tuberculosis. Infect Immun 2000;68:4477-84.

15. Daniel TM, Bernard BW. Mycobacterial antigens: a review of their isolation, chemistry and immunological properties. Microbiol Rev 1978;42:84-113.

16. Torres A, Juarez M, Cervantes R, Espitia C. Molecular analysis of Mycobacterium tuberculosis phosphate specific transport system in Mycobacterium smegmatis. Characterization of recombinant $38 \mathrm{kDa}$ (PstS-1). Microbial Pathogenesis 2001;30:289-97.

17. Samanich K, Keen M, Vissa V, Harder V, Spencer $\mathrm{J}$, Belisle $\mathrm{T}$ et al. Serodiagnostic potential of culture filtrate antigens of Mycobacterium tuberculosis. Clin Diag Lab Immun 2000;7:662-8.
18. Espitia C, González R, Mancilla R. A 38-kDa of Mycobacterium tuberculosis antigen associated with infection, its isolation and serologic evaluation. Clin Exp Immunol 1989;77:373-7.

19. Diagbouga S, Fumoux F, Zoubga A, Sanou PT, Marchal G. Inmunoblot analysis for serodiagnosis of tuberculosis using a 45-47 $\mathrm{kDa}$ antigen complex of Mycobacterium tuberculosis. Clin Diagn Lab Immunol 1997:4:334-8

20. Espitia C. and Mancilla R. Identification Isolation and partial characterization of Mycobacterium tuberculosis glycoproteins antigen. Clin Exp Immunol 1989;77:378-83.

21. Espitia C, Espinosa R, Saavedra R, Mancilla R, Romain F, Laqueyrerie $A$ et al. Antigenic and structural similarities between Mycobacterium tuberculosis 50/55 kDa and Mycobacterium bovis BCG 45/47 kDa antigens. Infec Immun 1995;63:580-4.

22. Raja A, Devi U, Ramalingam B, Brennan J. Immunoglobulin $G, A$, and $M$ responses in serum and circulating immune complexes elicited by the 16-kilodalton antigen of Mycobacterium tuberculosis. Clin Diagn Lab Immunol 2002;9:308-12.

23. Espitia C, Laclette JP, Mondragón-Palomino M, Amador A, Campuzano J, Martens A et al. The PE-PGRS glycine-rich proteins of Mycobacterium tuberculosis: a new family of fibronectin-binding proteins? Microbiol 1999;145:3487-95.

24. Singh X, Patibandla P, Chien Jr, Laal S. Antigens of Mycobacterium tuberculosis expressed during preclinical tuberculosis: serological immunodominance of proteins with repetitive amino acid sequences. Infect immune 2001;69:4185-91.

25. Espitia C, Sciutto E, Bottasso O, González-Amaro R, Hernández-Pando, Mancilla R. High antibody levels to the mycobacterial fibronectin-binding antigen of 30 $31 \mathrm{kDa}$ in tuberculosis and lepromatous leprosy. Clin Exp Immunol 1992;87:362-7.

26. Gennaro L. Inmunological diagnosis of tuberculosis. Clin Infect Dis 2000;30(Suppl.3):243-6.

27. Lyashchenko K, Manca C, Colangeli R, Heijbel A, Williams A, Gennaro M. Use of Mycobacterium tuberculosis complex-specific antigen cocktails for a skin test specific for tuberculosis. Infect Immun 1998; 66:3606-10.

28. Lyashchenko K, Mewa S, Singh M, Colangeli R, Gennaro M. A multi-antigen print immunoassay for the development of serological diagnosis of infectious diseases. J Immunol Methods 2000;242:91-100.

29. Houghton R, Lodes M, Dillon D, Reynolds L, Day C, McNeill P. Use of multiepitope polyproteins in serodiagnosis of active tuberculosis. Clin Diagn Lab Immunol 2002;9:883-91.

30. Espitia C, Mancilla R. The antigenic structure of Mycobacterium tuberculosis examined by immunoblot 
and ELISA. Influence of the age of culture and of the obtaining method on the composition of the antigenic extracts. Arch Invest Med (Méx) 1991;22:101-7.

31. Colangeli R, Heijbel A, Williams A, Manca C, Chan $\mathrm{J}$, Lyashchenko K. Three steps purification of lipopolysacharide-free polyhistidine tagged recombinant antigens of Mycobacterium tuberculosis. J Chromatogr B Biomed Sci Appl 1998;714:223-35.
32. Benjamin RG, Debanne SM, Ma Y, Daniel TM. Evaluation of mycobacterial antigens in an enzyme linked immunosorbent assay (ELISA) for the serodiagnosis of tuberculosis. J Med Microbiol 1984;18: 309-18.

33. Pérez C, Vargas M, Martinez L, Torres A, Villareal H. Results of a 12-month regime for drug-resistant pulmonary tuberculosis. Int J Tuberc Lung Dis 2002;6: 1102-9. 